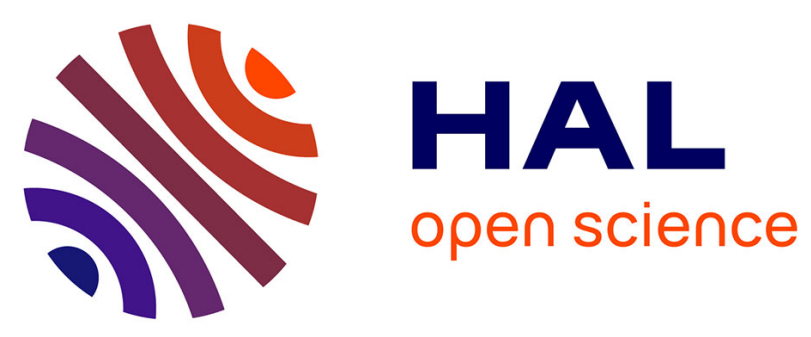

\title{
Note sur le post-Taylorisme
}

Christiane Barrier

\section{- To cite this version:}

Christiane Barrier. Note sur le post-Taylorisme. Sociologie du Travail, 1994, vol. 36 (n 3, juil. 1994), pp. 377-379. hal-00979720

\section{HAL Id: hal-00979720 \\ https://hal.science/hal-00979720}

Submitted on 24 May 2014

HAL is a multi-disciplinary open access archive for the deposit and dissemination of scientific research documents, whether they are published or not. The documents may come from teaching and research institutions in France or abroad, or from public or private research centers.
L'archive ouverte pluridisciplinaire HAL, est destinée au dépôt et à la diffusion de documents scientifiques de niveau recherche, publiés ou non, émanant des établissements d'enseignement et de recherche français ou étrangers, des laboratoires publics ou privés. 


\section{Christiane Barrier-Lynn}

\section{Note sur le post-taylorisme}

II y a plus de vingt ans que mon activite de recherche ne s'exerce pius dans le champ de la sociologie du travail. 11 m'arrive cependant de temps à autre de jeter un regard sur Sociologie du Travail. C'est ainsi que j'ai eu la curiosité de parcourir la livraison 1993. Les numéros 2 et 3 nappellent pas de commentaires de ma part sinon des compliments aux auteurs. En revanche le débat sur le post-taylorisme me laisse perplexe.

Me permettra-t-on d'ajouter à un débat déjà riche en opinions diverses - sinon divergentes - mes propres réactions inspirées par des pratiques de recherche que d'aucuns pourront trouver datées mais qui conservent néammoins leur validité?

Ma première surprise, à la lecture du débat sur le post-taylorisme vient de ce qu'un sujet aussi éculé ne rassemble pas un plus large consensus. J'impute ce qui m'apparâ̂t comme une insuffisance de consensus à certaines carences méthodologiques et épistémologiques. Le débat m'est apparu, à bien des égards, plus mondain que scientifique, dans la mesure où aucun critère ne permet de choisir parmi les propositions ou hypothèses avancées celles qui méritent d'être retenues pour valides et celles qui doivent être rejetées. Certes je ne mets pas en doute l'émergence de nouvelles formes d'organisation; mais si l'on entre dans les détails, les allées et venues entre propositions théoriques et observations ne semblent ni parfaitement maitrisées par les auteurs du "Dossier-Débat" ni vérifiables par d'autres.

Par exemple Veltz et Zarifian distinguent modèle d'organisation et lechnique d'organisation du travail mais n'expriment pas les liens entre les deux notions. Ils précisent que leur modèle «articule intimement les dimensions techniques, sociales et économiques d'un univers de production [...] le modèle d'organisation est fondamentalement ce qui réunit les dimensions sociales et les dimensions cognitives d'un univers de production [...].

En parlant de modèle, nous voulons également insister sur les discontinuités historiques qui caractérisent l'évolution de ces structures socio-cognitives». Cela dit, si l'on parle de modèle, c'est bien pour permettre de classer, abstraire les caractères essentiels des situations concrètes. Or à quels types de situations renvoient nos auteurs? A un panorama de l'industrie en Europe? dans les pays les plus industrialisés? dans les pays dits «de l'Est»? dans les 
pays d'Asie? dans le monde entier"? dans certaines branches d'activité particulières? Qui peut le dire? Pourtant it semble peu rigoureux d'amalgamer toutes les régions du monde et toutes les branches d'activité à l'intérieur de chaque région.

La définition du modè d'organisation avancée par Velz et Zarifan, pour importante qu'elle soit dans la construction du débat, tel qu'il a été conçu, me laisse une grande impression de malaise épistémologique. Un modele ne prend de sens que s'il résume, interprète, explique un grand nombre de données factuelles. En l'absence de référence à des données méthodiquenent classées et ordonnées, un modèle peut sembler parfatement abitraire é évoquer une pseudo-théorie plutot qu une théorie validement fondée. Le mot même de modele ne devrain-il pas être réservé aux modeles logico-mathématiques? ou du moins aux démarches conceptuelles qui s'inspirent de la fonction logique des modèles mathématiques? Je ne suis done pas certaine que le recours au modele de Velo et Zarifian nouts éclaire vraiment sur les changements réels en cours.

Jean-Louis Laville, hii, nous dit s'appuyer sur 27 études de cas. Son discours peut paraitre plus élayé, plus fondé danss la réalité. Je regretre cependant que son choix d'entreprises ne recouve pas une plus grande variété de branches; je regrelle que l'indusirie chinique, les rafineries, les centrales électriques, la pétrochimie... ne fïgurent pas dans ses monographies, alor's qu'il a retenu neuf entreprises métalkurgiques et six ans dans l'électro-ménager.

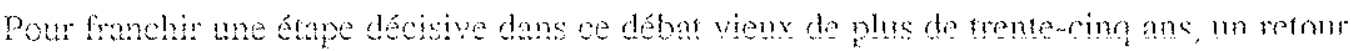
à de vastes enquêtes quantitatives représentatives permettrait réellement de saisir la pluralité des tendances à l'axuve dans nos diverses sociécés el de départager à juste titre les opinions qui s'aftrontent dans leur analyse. Ce type d'enquête fournirait, me semble-i-il, de meilleurs moyens de raisonner, en se fondant sur des critères contrôlables, dans certaines limites de confiance. Par crainte -- devenue peut-être excessive -- du déterminisme technologique, l'influence des facteurs techniques sur les nouvelles formes d'organisation, m'a paru sous-estimée.

Je voudrais rappeller que ces facteurs interviennent tout de même dans la disparition de facto du taylorisme. Il suffit de rappeler que dans la chimie, la pétro-chimie, les rathneries, les centrales électriques -. industries qui produisent des licquides, des gaz et un "produit" impalpable, l'électricité, qui ne recourent ni aux outils ni au travail en miettes - le taylorisme n'a jamais pu regle la division et l'organisation du travil. fesentiel des tâches productives, dans ces branches, consiste en tâches de surveillance associés à une gamme d'interventions en réponse à diftérents signaux, le plus souvent lumineux ou sonores. Ce type d'interventions suppose de la part des "surveillants" une capacité d'initiative et d'autonomie variable suivant les postes. Leur niveau de responsabilité peut être apprécié par la gravité et le nombre d'incidents critiques qu'une réaction non pertinente déclenche en aval du flux de production.

De plus, les tâches directement liées à la production ne sont pas forcément les plus nombreuses dans ce type d'industries. Dans les premières centrales nucléaires françaises, les services d'entretien et du contrôle technique comptajent déjà chacun plus d'agents que ceux de la production. Dans l'ensemble de l'EDF, les services de recherche, du transpon de l'énergie, de sa distribution... cumulaient des effectifs sans commune mesure avec ceux de la production. A l'intérieur même des centrales, l'organisation du travail dans les services d'entretien, et également dans ceux du contrôle technique, ne pouvait être assimilée à ce qu'elle était dans les services directement productifs, elle-même sans parenté avec l'organisation du travail sur les chânes d'assemblage ou dans les laminoirs, par exemple, puisqu'elle était caractérisée par le pilotage à distance à partir d'une seule salle de contrôle. Ces différences de base dans les modes de travail sont passées sous silence dans le dossier-débat 
gui progresse par amalgame d'on ne sait quoi avec on se sait quoi. Ce genre d'oubli ou d'amalgame le fait paraître reposer sur des abstactions arbitraires et hi donne un caractère vague pour ne pas dire « fumeux».

Une vole d'analyse plus claire aurait pu consister à classer les modes d'organisation concrète du travail en fonction de plusieurs criteres dont le niveau d'automatisation des éablissements, ou des entreprises, défini en s'inspirant par exemple de l'échelle des niveaux dautomatisation établie par James Bright il y a déjà 36 ans.

Le débat n'auratit-il pas gagné en clarté si l'on avaił raisonné en distinguant:

-.- Les branches d'activité où perdure le taylorisme, celles d'où il a été complètement évacué ou ne s'est jamais imposé, celles où il s'infilite.

-.- Les régions et pays d'ou il disparât (pays les plus industrialisés), ceux ou il se stabilise (pays de l'Est européen? ) ceux où les pays les plus industrialisés l'exportent: industries délocalisées dans les pays à industries de main-d'cuvre.

l.e déba n'aurat-il pass également gagné à être situé dans celui sur la crise mondiale actuelle? Denis Clere et Alain Lipiet (1985) attribuent, pou une large part, lá crise économique à celle du taylorisme-fordisme. Cette hypothèse (taxée de "réformiste» par les auteurs marxistes) ne mérite-t-elle pas d'être explorée plus à fond? En tout cals, elle ouvre des perspectives et requiert que l'on cherche à l'invalider ou à la retenir. L'ère da post-taytorisme doit.elle être celle d'une crise indéfinie?

Christiane BARRYRR-LYYNN

Conservatoire national des Arts et Métiers

(CNAM-CNRS),

10, me Saint-Roch,

75001 Paris

\section{BHBLIOGRAPIME}

BRGHT 3. R. (1958), Amomation and Managemem, Boston, Harvard School of Business Administration, $270 \mathrm{p}$.

Chire D., LIPHitz A. el SATRE-Busson J. (1985), La Crise, Paris, Syros-Allematives, 167 p. 